\title{
Dosimetric Characteristics of 6 MV Modified Beams by Physical Wedges of a Siemens Linear Accelerator
}

\author{
Mansour Zabihzadeh ${ }^{1,2,3}$, Mohammad Javad Tahmasebi Birgani ${ }^{1,2}$, Mojtaba \\ Hoseini-Ghahfarokhi ${ }^{1,3}$, Sholeh Arvandi ${ }^{2}$, Seyed Mohammad Hoseini ${ }^{2}$, Mahbube \\ Fadaei $^{1 *}$
}

\begin{abstract}
Physical wedges still can be used as missing tissue compensators or filters to alter the shape of isodose curves in a target volume to reach an optimal radiotherapy plan without creating a hotspot. The aim of this study was to investigate the dosimetric properties of physical wedges filters such as off-axis photon fluence, photon spectrum, output factor and half value layer. The photon beam quality of a 6 MV Primus Siemens modified by 150 and 450 physical wedges was studied with BEAMnrc Monte Carlo (MC) code. The calculated present depth dose and dose profile curves for open and wedged photon beam were in good agreement with the measurements. Increase of wedge angle increased the beam hardening and this effect was more pronounced at the heal region. Using such an accurate MC model to determine of wedge factors and implementation of it as a calculation algorithm in the future treatment planning systems is recommended.
\end{abstract}

Keywords: Beam hardening - Monte Carlo calculation - physical wedge filter - radiotherapy

Asian Pac J Cancer Prev, 17 (4), 1685-1689

\section{Introduction}

Assuming uniform clonogenic cell distribution in the tumor volume, the aim of conformal radiotherapy techniques is to obtain of high local tumor control probability (TCP) and keeping the normal tissue complication probability (NTCP) as low as possible (Muren et al., 2001; Khan, 2014). Therefore, the International Commission on Radiation Units and Measurements(ICRU) recommends a limit of minimum and maximum dose in the planning process of target volume within $-5 \%$ and $+7 \%$ of the prescribed dose to reach a high and uniform dose distribution in the target volume and minimal possible dose in any organ at risk (ICRU: Report 50; 1993).

The physical wedge filters (PWs) are made from steel, lead or tungsten alloys and modulate the photon intensity across the beam. They are located in beam pathway to cause substantial attenuation in the intensity of photon beams and increase the average energy of transferred beam. Due to the irregular body contour and tumor volume, the beam modifiers such as the PWs are still widely used in external beam radiation therapy to improve the dose uniformity in the planning target volume (Ahmad et al., 2010; Njeh, 2015) beside the progressive techniques such as enhanced dynamic wedge (Kowalik et al., 2013). It can be used as a missing tissue compensator as well as to shift the isodose pattern toward its thinner edge at a target volume without creating a hotspot (Muren et al., 2009; Vinagre et al., 2009). Determination of the changes value in beam quality and dose distribution curves due to presence of PW across the radiation field is critical to calculate of dose distribution in the treatment planning systems (TPS) (Miften et al., 2000; Petrovic et al., 2010; Muhammad et al., 2011). The dosimetric properties of the hardened photon beam by PW filters may be varied between different linear accelerators (Linac) and even for the same model. Therefore the dosimetric parameters of wedge filters must be exactly known for each Linac, individually. Directly measuring of these parameters is difficult, time consuming and tedious. In the recent years, Monte Carlo (MC) calculation methods are well known to accurately survey of radiation beams used to diagnostic and therapeutic applications (Verhaegen et al., 1999; Shih et al., 2001).

In this study the x-ray beam quality indexes such as photon spectrum, mean energy, half-value layers (HVL) beside the percent depth dose (PDD) and dose profile curves for $6 \mathrm{MV}$-Siemens Linac were studied in the presence of 150 and 450 PWs using BEAMnrc and DOSXYZnrc packages.

\section{Materials and Methods}

\section{Measurements}

The measurements were performed for Siemens Primus

${ }^{1}$ Department of Medical Physics, Faculty of Medicine, ${ }^{2}$ Departments of Clinical Oncology, Golestan Hospital, ${ }^{3}$ Student Research Committee, Ahvaz Jundishapur University of Medical Sciences, Ahvaz, Iran*For correspondence: mahbubefadaei@ymail.com 
Linac $6 \mathrm{MV}$ photon beam according to recommendations of IAEA protocol, TRS-398 (Andreo et al., 2000). Measurements of the PW factors were carried out using $0.125 \mathrm{~cm} 3$ Farmer type ionization chamber with DOSE1 electrometer (FC65G, Scanditronix-Wellhofer, Germany) at the depth of $10 \mathrm{~cm}$ with source to surface distance (SSD) of $100 \mathrm{~cm}$. The PDD and dose profile curves for open and PW field of $10 \times 10 \mathrm{~cm}^{2}$ were measured in 50 $\mathrm{cm}^{3}$ PTW-Blue water phantom and processed by dosimetry software RFAplus (Version 5.2, Scanditronix-Wellhofer, Germany). Each measurements were repeated three times with precision of $0.2 \%$.

\section{MC simulation}

MC simulations were performed by EGSnrc MC code based user codes of BEAMnrc and DOSXYZnrc (Rogers et al., 2003). The dimensions and materials of different components of Linac head were built using data from the vendor. A number of component modules (CMs) including of SLAB, FLATFILT, CHAMBER, MIRROR, JAWS and PIRAMIDS were used to modeling of exit window, target, primary collimator \& flattening filter, monitoring chambers, mirror, jaws and PWs filter, respectively. In all calculations, ECUT (lower energy threshold for electron transport), similar to AE (threshold energy for electron creation) and PCUT (lower energy threshold for photon transport), similar to AP (threshold energy for photon creation) were $0.700 \mathrm{MeV}$ and $0.01 \mathrm{MeV}$, respectively. Directional bremsstrahlung splitting (DBS) technique with splitting number of 1000 was used as one of the variance reduction method. In addition, electron range reduction method with ESAVE_GLOBAL $=2 \mathrm{MeV}$ was applied in simulation of the whole head with the exception of the target CM where ESAVE_GLOBAL was $0.7 \mathrm{MeV}$. All other EGSnrc parameters were set to their default values.

The modes of open field (without a PW) and $15^{\circ}$ or $45^{\circ} \mathrm{PW}$ modulated fields were evaluated. One of the phase space files was scored in a plan perpendicular to the beam axis which was located just below the down surface of $\mathrm{X}$-jaws. Two other score plans were defined to generate phase space files at surface of the phantom for both open and wedge-filtered fields. Two steel wedges of $15^{\circ}$ or $45^{\circ}$ with non-linear profiles were located at $40.2 \mathrm{~cm}$ from the target under the X-jaws. Electron histories of $1.5 \times 10^{8}$ were run to collect about 300 million particles in the first phase space file. The particles in each phase space were recycled several times in order to achieve an interested statistical uncertainty.

The PDD and dose profiles were calculated in DOSXYZnrc using a water phantom of $50 \times 50 \times 50 \mathrm{~cm}^{3}$ positioned at SSD of $100 \mathrm{~cm}$ for field size of $10 \times 10$ $\mathrm{cm} 2$. The voxel size with dimensions of $1 \times 1 \mathrm{~cm}^{2}$ in the lateral directions and $0.25 \mathrm{~cm}$ resolution in depth were considered for PDD calculations. The lateral dose profiles were calculated for depth of $10 \mathrm{~cm}$ with $0.2 \mathrm{~cm}$ resolution in $\mathrm{X}$ or $\mathrm{Y}$ direction and $1 \mathrm{~cm}$ in the remaining two directions. Number of histories in DOSXYZnrc was selected 4 billion particles to reach the statistical uncertainties $<1 \%$ in all MC calculations. Characteristic of photons like spatial fluence, energy fluence and mean energy was extracted from the calculated phase space at phantom surface using the BEAMDP interface. The photon fluences vs. lateral off-axis distances were scored in $12.5 \times 0.5 \mathrm{~cm} 2$ rectangular bins across the field. The photon energy spectra were evaluated by scoring the photon energy distribution in rectangular bins with resolution of $1 \mathrm{~cm}$ along the angled side of $\mathrm{PW}$ in toe, center and heel regions.

The off-axis variation of output factor (the ratio of absorbed doses at a reference depth with and without wedge) was investigated for both $\mathrm{x}$ and $\mathrm{y}$ directions at a depth of $10 \mathrm{~cm}$ and SSD of $100 \mathrm{~cm}$. Spectral distribution (energy fluence in each energy interval) contains valuable data about the radiation beam, especially beam quality which can be represented by several indexes such as HVL, TPR20,10, etc. Although the measurement of spectral distribution can be so demanding, MC codes can easily produce it. Therefore, beam hardening effect was investigated at toe, center and heel regions using three $1 \times 12.5 \mathrm{~cm} 2$ bins across the field in the form of half value layer (HVL) that defined as the thickness, $t$, of water needed to attenuate the in-air collision Kerma, Kc, to half of its measure from when no water was present (equation 1). The summation was performed over 200 equidistant energy bins with fluence, $\frac{\Delta \phi}{\Delta \mathrm{E}_{\mathrm{i}}}$, mid-energy, $E_{i}$, and energy width, $\Delta E_{i}$. The linear attenuation coefficient, $\mu$, and the mass energy absorption coefficient, $\mu_{\mathrm{en}} / \mathrm{p}$, for each energy bin were extracted from the XAAMDI database (Hubbell et al., 1995).

$$
\frac{\mathrm{K}_{c(t)}}{\mathrm{K}_{c(0)}}=\frac{\sum_{n=i}^{N}=\frac{\Delta \phi}{\Delta \mathrm{E}} E_{i} \frac{\mu_{\mathrm{en}, \mathrm{air}}}{p}\left(E_{i}\right) e\left[-\mu\left(E_{i}\right)_{\text {absorber }}\right) \Delta E_{i}}{\sum_{n=i}^{N}=\frac{\Delta \phi}{\Delta \mathrm{E}} E_{i} \frac{\mu_{\mathrm{en}, \text { air }}}{p}\left(E_{i}\right) \Delta E_{i}}=\frac{1}{2}
$$

All needed data for solving of equation 1 were extracted from XAAMDI database and from MC calculations and processed by homemade program in MATLAB software.

All simulation processes was done in parallel mode on Ubuntu operating system installed on a computer, equipped with 21 cores of Intel Xeon® CPU X7560@2.27 $\mathrm{GHz}$ and $16 \mathrm{~GB}$ of RAM.

\section{Results}

\section{Benchmark of simulated Linac head Open field}

An tuned incident electron beam with the mean energy of $6.2 \mathrm{MeV}$, the Gaussian energy spread with FWHM=1 $\mathrm{MeV}$ and the spatial FWHM of $1 \mathrm{~mm}$ resulted a good agreement between the measured and calculated data. The agreement between measurements and calculations (Figure 1) were within 1\% for depth dose profile beyond the depth of maximum dose and for the lateral profile inside the field.

\section{Physical wedged fields}

In order to validate the MC simulated PWs, the calculated PDD and lateral dose profiles of $15^{\circ}$ and $45^{\circ}$ physical wedged fields were compared to measurements. Only in-line dose profiles (in Y direction) are depicted in Figure 2. The MC calculations agreed well with the measurements within $2 \%$ inside the field for both of the wedges. 


\section{Off-axis photon fluences}

The off-axis photon fluences of open and $15^{\circ}$ and $45^{\circ}$ physical wedged fields are calculated in resolution of 5 $\mathrm{mm}$ as shown in Figure 3. It is clear from Figure 3 that photon fluence is nearly uniform across the field at open field while for wedged field decreases from the toe to the heel region of the wedge. This effect is more significant at larger wedge angles.

\section{Photon energy fluence}

The calculated spectral distributions of $6 \mathrm{MV}$ photons from $10 \times 10 \mathrm{~cm}^{2}$ at $\mathrm{SSD}=100 \mathrm{~cm}$ for toe, central and heal regions of open and $15^{\circ}$ and $45^{\circ}$ physical wedged

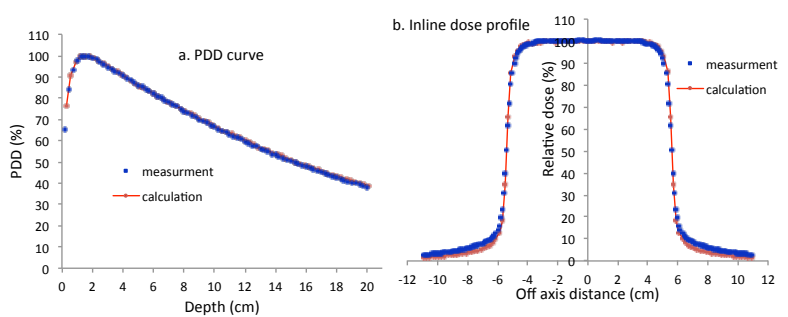

Figure 1. Comparisons of Calculated and Measured. a) PDD and $b$ ) Lateral dose profile for SSD of $100 \mathrm{~cm}$ and open field of $10 \times 10 \mathrm{~cm}^{2}$. The PDD was normalized to 100 at maximum dose depth. Lateral dose profile in depth of $10 \mathrm{~cm}$ was normalized to its central voxel value

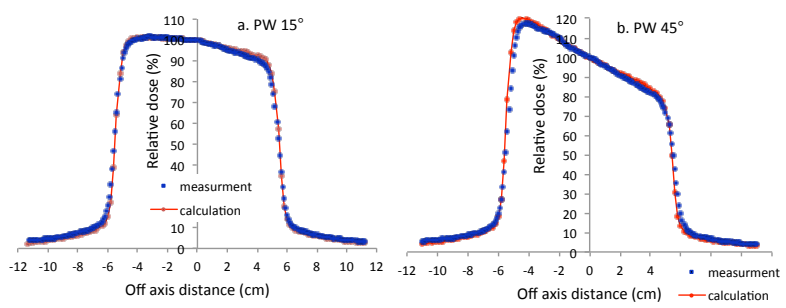

Figure 2. Comparison of the MC calculated and experimentally measured in-line dose profiles for a). $15^{\circ}$ and $\left.\mathrm{b}\right) .4^{\circ}$ physical wedged fields. Lateral dose profile in depth of $10 \mathrm{~cm}$ was normalized to its central axis value as $100 \%$

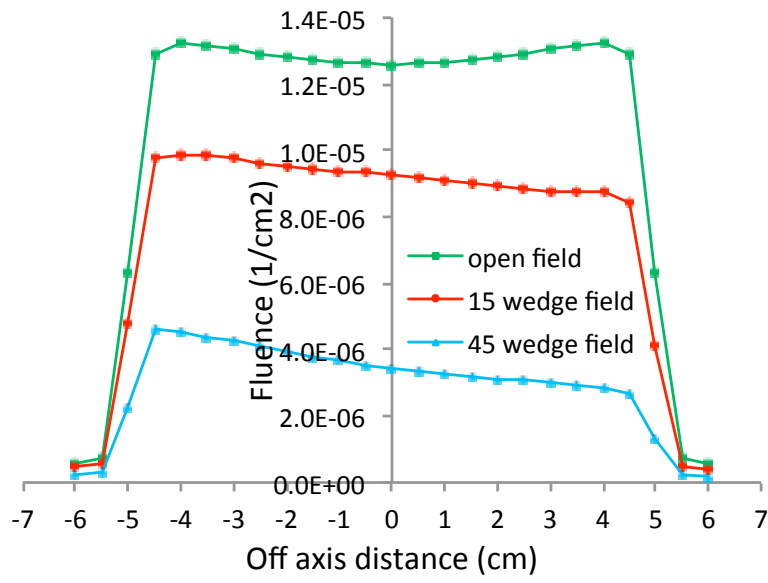

Figure 3. In-line Photon Fluences for Open and $15^{\circ}$ and $4^{\circ}$ Physical Wedged Fields of $10 \times 10 \mathrm{~cm}^{2}$ at $\mathrm{SSD}=100$ cm. The Photon fluences are normalized per incident electron on target. fields are presented in Figure 4. From Figure 4, it can be dedicated that energy fluence for PWs decrease across the wedge angled direction from the toe to the heal region compared to open field. This effect is more significant for the $45^{\circ}$ wedge.

Output (wedge) factor

From Figure 5.a it can be found that the in-line output

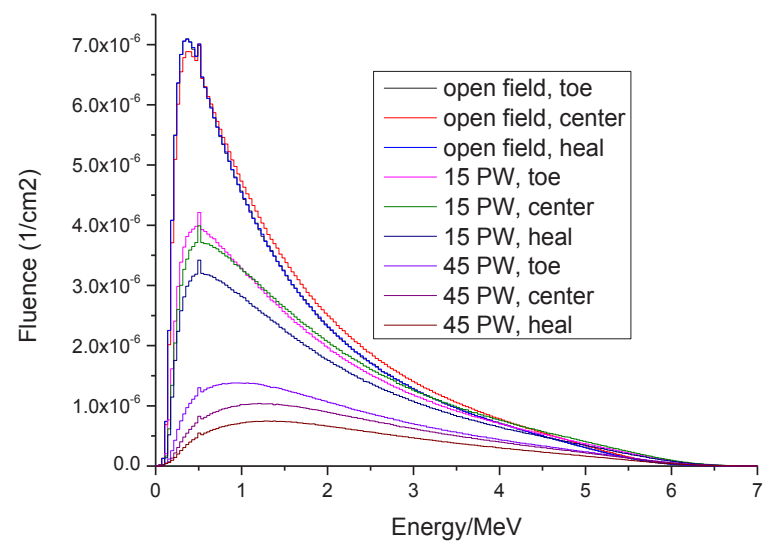

Figure 4. The Photon Spectral Distributions of $6 \mathrm{MV}$ Photon in the Toe, Central and Heal Regions of Open and $15^{\circ}$ and $45^{\circ}$ Physical Wedged Fields for $10 \times 10$ $\mathbf{c m}^{2}$ field at $\mathbf{S S D}=\mathbf{1 0 0} \mathbf{~ c m}$. The Photon energy fluences are normalized per incident electron on target
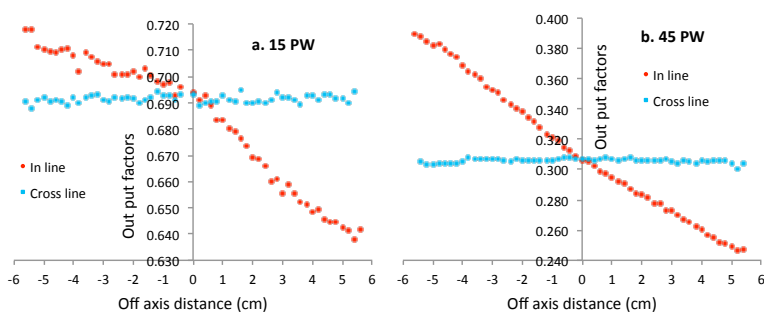

Figure 5. The variation of in-line and cross-line output factors across the beam line a) $15^{\circ}$ wedge and b) $45^{\circ}$ wedge; for $6 \mathrm{MV}$ photon beam in $10 \times 10 \mathrm{~cm}^{2}$ field size at $\mathrm{SSD}=100 \mathrm{~cm}$

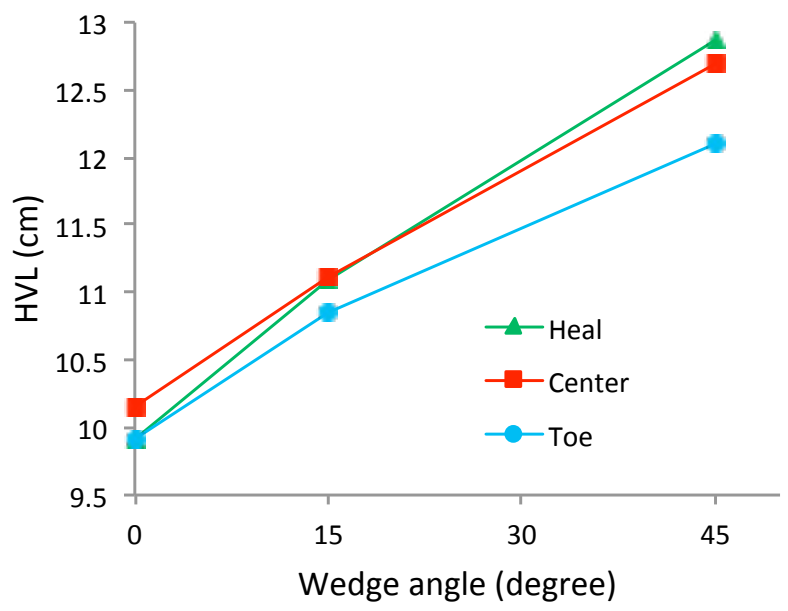

Figure 6. The HVL changes (in term of water, cm) with wedge angles at the toe, central and heal regions of $15^{\circ}$ and $45^{\circ} \mathrm{PWs}$ 
factors decrease across the field from the toe to the heal region and this change is more pronounced for $45^{\circ}$ wedge. Additionally, it is clear that output factor is not changed along the cross-line direction (Figure 5.b).

\section{Half Value Layer (HVL)}

The beam hardening effects of the $15^{\circ}$ and $45^{\circ}$ PWs in term of HVL are presented in Figure 6. HVLs (absorber=water, $\mathrm{cm}$ ) increase from the toe to the heal region across the beam for each of $15^{\circ}$ and $45^{\circ}$ physical wedged fields. The maximum alternation of $\mathrm{HVL}$ $29.77 \%$, was obtained at the heal region due to more beam hardening effect. However, there was no significant difference between the central and heal parts of $15^{\circ} \mathrm{PW}$.

\section{Discussion}

In this study the maximum relative error of calculations was better than $1 \%$ at in-side and out-side of the field. The comparison of PDD and dose profile curves for open field without PWs show a good agreement between MC calculated results and measurements. The source of the negligible difference in out of field of dose profile may be caused by inaccurate provided data by the vendor or nonideal tuning of incident electron beam parameters due to its time consuming. For the wedged fields this agreement was better than $2 \%$. The slight discrepancies may be related to little uncertainties in the real steel composition. These agreements are comparable with reported results by other groups (Zhu et al., 2000; Shih et al., 2001; Attalla et al., 2010).

According to Figure 3, PWs reduce photon fluence entirely due to attenuation effect of PW and this reduction is higher by increasing of wedge angle. Across the beam line, the reduction of photon fluence increase from the toe to the heal region of PW due to the higher thickness of heal part than toe along the photon beam path. The average energy of photon were 1.62, 1.57 and 1.62 MV for open field, 1.85, 1.92 and 1.91 MV for $15^{\circ} \mathrm{PW}$ and $2.16,2.31$ and 2.35 MV for $45^{\circ} \mathrm{PW}$, respectively (Figure 4). For toe, central and heal regions of the beam, the mean calculated energy shifts to higher energy with increase in wedge angle that means the presence of wedge in beam line decreases the lower energy components of beam more strongly than the higher energy photons. This is more significant for the heel region due to more thickness of steel wedge filter. These results are in consistence with other reported data (Verhaegen and Das, 1999; Zhu et al., 2000).

As shown in Figure 5, the output factors changed across the in-line direction from 0.638 to 0.718 and from 0.246 to 0.383 for $15^{\circ}$ and $45^{\circ} \mathrm{PWs}$, respectively. The averaged value of in-line output factor was 0.313 and 0.683 for $15^{\circ}$ and $45^{\circ} \mathrm{PWs}$, respectively. These output variations are not considered in treatment planning systems (TPS) or during manual calculation to determination of dose distribution. Our calculated in-line output factors on the central axis in depth of $10 \mathrm{~cm}$ and $10 \times 10 \mathrm{~cm}^{2}$ field size at SSD=100 were 0.693 and 0.307 , that are in a good agreement with measured data of 0.675 and 0.312 for $15^{\circ}$ and $45^{\circ}$ PWs, respectively. As expected, the cross-line output factors (square dots in Figure 5) of wedged fields show no meaningful variations $v s$. off-axis distance due to the same attenuating thickness of wedge across the cross-line direction of photon beam.

As can be seen from Figure 6, the HVL increase from the toe to the heal regions for each wedges; $2.12 \%$ and $6.28 \%$ for $15^{\circ}$ and $45^{\circ} \mathrm{PWs}$, respectively. In the central region, in spite of the larger thickness of $45^{\circ} \mathrm{PW}$ than $15^{\circ} \mathrm{PW}$, the HVLs of $15^{\circ}$ and $45^{\circ} \mathrm{PWs}$ are same. It can be explained that the more production of scattered photons (due to the larger scattering mass in case of $45^{\circ} \mathrm{PW}$ ) may compensate the hardening effect. Dependence of mean photon energy to the wedge angle for other Linac models such as Varian has been well documented by Geraily, et al. (2014). For the open field the HVL of the center region is $2.42 \%$ larger than the toe and the heal regions; It can be caused by attenuation properties of the Gaussian shape flattening filter located in Linac head that has more fluence and energy attenuating powers in its central part. This effect is clear from off-axis fluence curve of open field in Figure 3.

In conclusion our MC modeling of physical wedge dosimetric properties are in good agreement with measurements. The results indicate that the presence of a wedge in beam line causes beam hardening. Increase of wedge angle increases the beam hardening and this effect is more pronounced at the heal region of PWs. Using of such accurate MC model to determine of wedge factors and implementation of it as a calculation algorithm in treatment planning system (TPS) is recommended.

\section{Acknowledgements}

This study was Funded by the research and technology deputy of Ahvaz Jundishapur University of Medical Sciences \& Arvand international University of Medical Sciences, Ahvaz, Iran.

\section{References}

Ahmad M, Hussain A, Muhammad W, et al (2010). Studying wedge factors and beam profiles for physical and enhanced dynamic wedges. J Med Physics, 35, 33-41.

Andreo P, Burns DT, Hohlfeld K, et al (2000). IAEA ,TRS-398: Absorbed dose determination in external beam radiotherapy: An International code of practice for dosimetry based on standards of absorbed dose to water. IAEA: international atomic energy agency. Vienna, 10, 46-80.

Attalla EM, Abo-Elenein HS, Ammar H, et al (2010). Comparison of dosimetric characteristics of Siemens virtual and physical wedges for ONCOR linear accelerator. J Med Physics, 35, 164-9.

Geraily GH, Mirzapour M, Mahdavi S.R, et al (2014). Monte Carlo study on beam hardening effect of physical wedges. Int J Radiat Res, 12, 249-56.

Hubbell JH, Seltzer SM (1995). Tables of x-ray mass attenuation coefficients and mass energy absorbtion coefficients from $1 \mathrm{keV}$ to $20 \mathrm{keV}$ for elements $\mathrm{Z}=1$ to 92 and 48 additional substances of dosimetric interest. NISTIR, $\mathbf{5 6 3 2}$.

IAEA, report: TRS-398 (2000). Absorbed dose determination in external beam radiotherapy. Veina, International atomic energy agency.

ICRU: Report -50 (1993). Prescribing, recording and reporting photon beam therapy. Bethesda, MD: International 
Commission on Radiation Units and Measurements.

Kowalik A, Litoborski M (2013). Multienergetic verification of dynamic wedge angles in medical accelerators using multichannel linear array. Reports Practical Oncol Radiotherapy, 18, 220-34.

Miften M, Zhu XR, Takahashi K, et al (2000). Implementation and verification of virtual wedge in a three-dimensional radiotherapy planning system. Med Physics, 27, 1635-43.

Muhammad W, Maqbool M, Shahid M, et al (2011). Assessment of computerized treatment planning system accuracy in calculating wedge factors of physical wedged fields for 6 MV photon beams. Physica Med, 27, 135-43.

Muren LP, Hafslund R, Gustafsson A, et al (2001). Partially wedged beams improve radiotherapy treatment of urinary bladder cancer. Radiotherapy Oncol, 59, 21-30.

Njeh CF (2015). Enhanced dynamic wedge output factors for Varian $2300 \mathrm{CD}$ and the case for a reference database. $J$ Applied Clinical Med Physics, 16, 5498.

Petrovic B, Grzadziel A, Rutonjski L, et al (2010). Linear array measurements of enhanced dynamic wedge and treatment planning system (TPS) calculation for $15 \mathrm{MV}$ photon beam and comparison with electronic portal imaging device (EPID) measurements. Radiol Oncol, 44, 199-206.

Rogers DWO, Ma C-M, Walters B, et al (2003). BEAMnrc User Manual. NRCC Report PIRS-509 (National Research Council of Canada), Ottawa, Ontario, Canada.

Shih R, Li XA, Chu JC (2001). Dynamic wedge versus physical wedge: a Monte Carlo study. Medical Physics, 28, 612-9.

Verhaegen F, Das IJ (1999). Monte Carlo modelling of a virtual wedge. Physics Med Biol, 44, 251-9.

Vinagre FL, Simoes PC, Rachinhas PJ (2009). Omni-wedge technique for increased dose homogeneity in head and neck radiotherapy. Physica Med, 25, 154-9.

Zhu XR, Gillin MT, Jursinic PA, et al (2000). Comparison of dosimetric characteristics of Siemens virtual and physical wedges. Medical Physics, 27, 2267-77. 